Volume 10, No.3, May - June 2021

International Journal of Advanced Trends in Computer Science and Engineering

Available Online at http://www.warse.org/IJATCSE/static/pdf/file/ijatcse591032021.pdf

https://doi.org/10.30534/ijatcse/2021/591032021

\title{
VANET routing Protocols :Implementation and Analysis Using NS3 and SUMO
}

\author{
Maria Jan', Salman Afsar ${ }^{2}$, Ahmed Mateen ${ }^{3}$, Muhammad Qasim Yasin ${ }^{4}$, Bilal Safdar ${ }^{5}$ and Abdul Rehman \\ 1,2,3,5 Department of Computer Science, University of Agriculture Faisalabad. \\ ${ }^{4}$ Computer Science Department, National University of Modern Languages (NUML), Faisalabad. \\ ${ }^{6}$ College of Intelligence and Computing, Tianjin University, China. \\ Corresponding Author Email: ahmedmatin@ hotmail.com
}

\begin{abstract}
The emerging new form of mobile adhoc network VANET Network is exemplified by fast and drastic changes in topology over a shorter period of time. These two features have a significant impact on the efficiency of routing protocols used in these networks. The evaluation of various VANET routing protocols provides great feedback on the efficiency of the network. The efficacy of AODV, OLSR, DSDV and DSR protocols is evaluated in this analysis, within the VANET intersection. The movement patterns of vehicles are generated with SUMO and the simulator is NS3. Considering VANET's high velocity, frequent disassociation and highly evolving topology, the major challenge is to create a routing protocol that is more appropriate to efficiently routing packet to their end destination. System of measurement such as power consumption, packet distribution factor, average performance as well as maximum end-to-end delay being calculated.
\end{abstract}

Key words: VANET, Ad-hoc Network, AODV, DSDV, OLSR, NS3, SUMO

\section{INTRODUCTION}

A subdivision of Mobile Ad-Hoc Networks (VANET) has become, as a collection of mobile Nodes, a collection knowledge as On-Board Units (OBUs) for the purpose of capturing, transmitting, downloading, or sending information to certain additional nodes. VANES (VOHCNetworks Vehicle-ad-hoc networks).[1].In recent times, VANET also brought a large amount several studies that investigated particularly $\mathrm{V} 2 \mathrm{~V}$ (vehicle-to-vehicle) as well as V2IV (vehicle-to-vehicle) but also V2II in diverse locations including such protection, service quality, Network Layer as well as others (vehicle-toInfrastructure)Communication systems Inside[2].

The layout including its communication systems as well as its protocols are managed by the other mobile ad hoc networks (MANETs), VANET has its own distinctive properties[2], [3]. VANET's exclusive features involves High dynamic topology: Determines the speed in vehicles, that topology of VANET outright violence.

Different communication environments: That travel times vary throughout urban to remote regions that are easy throughout country places yet challenging for urban areas due towards barriers such as the construction of trees as well as others[4].

Network variable density: VANET's traffic volume is not quite the same throughout the day and for certain sorts of situations.

Frequently disconnected network: VANETs would not have continuous connectivity because of the fast traffic flow.

Hard delay limitations: Several VANET frameworks maintain a specific delay due to large bandwidths.

SUMO is also a traffic simulator for traffic layout and road design optimization. An effort should be made with interface SUMO through NS2. Throughout this connection, we has used SUMO simulator for identify the functionality of vehicle traffic sensor nodes including[5] its influence on the quality through VANETs as well as to interface that to NS3 for determine the obtained results.

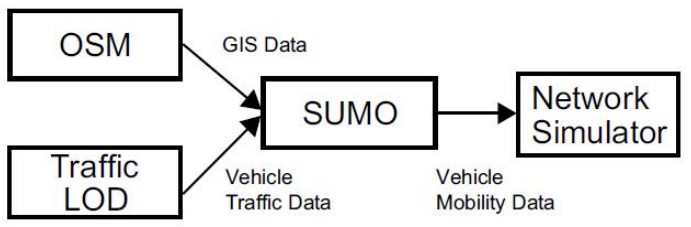

Figure 1: SUMO Classification

\section{DSDV (Destination-Sequenced Distance Vector):}

DSDV seems to be a proactive routing protocol where every other node maintains their sequence number throughout terms of maintaining its route throughout all stages between each network destination, however if the routes become technically inaccessible[6].Along with addition to the advantages in DSDV, including certain flexibility, that unlimited loop but no delay time triggered by that of the exploration including its path. Due to the repeated changes induced by that of the extremely modifiable topology including its networks, this same DSDV always benefits through overhead[7].

Optimized Link Stat Routing (OLSR):

Throughout OLSR, increasing sensor node anrreq packet frequently. That empowers a broad information about the network configuration to ever be built according to each link. That Multiple Link. Signal repeaters (MPR) principle is used by[8] OLSR for something like the transmitting of congestion control that decreases by only using selected nodes with overhead flood mitigation congestion which enhance that broadcasting method. 


\section{AODV:}

Within AODV, on some request routes get established whereas those are in use become preserved. Throughout this scenario, at just the start of class, an incremental delay would be required to check for such a route. Through AODV, a cluster head would transmit a request packet transmission RREQ to most of its peers that find out the route to the destination, well its peers will relay their RREQ towards their peers etc. until it receives the request or really any destination nodes which has new information about route to both the actions that will lead. Throughout this situation, after sustainable operation an RREP, that access point addresses[9]-[11].

\section{Dynamic Source Routing (DSR):}

Dynamic Source Routing (DSR) is a protocol based on the so-called source routing scheme for wireless networking. It is like AODV when a computer asks for a route on request. Unless each intermediate node's address ID is added to the portable packet list by the routing request packet. A route response message containing the statements established in the route request is generated by the intent node and sent through a path back to the source. Maintenance of the pathway in the DSR is carried out through node expectations towards check that the next node has successfully received a shipment.[12].

\section{Protocols for Routing Within Vanets:}

VANET routing protocols influence the way remote nodes (vehicles) detour information that share necessary data over a large period of time.Figure 2shows the protocol classification.

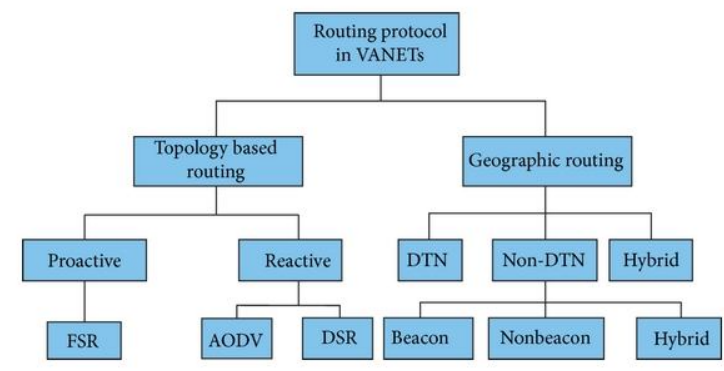

Figure 2: Ad-hoc Routing Protocols Classification

Individuals can be subdivided in to the 4 categories.

Topology-based routing protocols:

The connection information that resides within the network is often used in topology-based communication algorithms to transfer data from source to destination. The protocol group could be divided into 3 major types: reactive or on request protocols. AODV, constructive protocols as well as OLSR in addition DSDV or tabledriven protocol, also hybrid protocols like ZRP[13].

Position-based routing protocols:Such protocols require access to something like a tracking system including a GPS (Global Position System) which delivers information about the major location for vehicles on the route. These data are required in terms of deciding with Packet level of routes towards its eventual disposal.In fact, it is possible for the Delay Tolerant Network (DTN), Non-Delay Tolerant Network, or Position-based hybrid routing to be separated by 3 forms of position-based routing. [14], [15].

\section{Multicast-based routing protocols:}

The node can send throughout multi - hop routing protocols System uses inter communication, from either a particular source to any and all high bandwidth users. Such protocols could be categorized into 3 groups, particularly regarding protocols predicated around geocast, Mobicast as well as cluster[16].

\section{Broadcast-Based Protocols for Routing:}

The request is transmitted throughout webcast routing protocols. By a specific source to those network vehicles. VANET typically uses these protocols that communicate vehicle speed, traffic, climate including emergency events amongst vehicles[9], [15].

\section{LITERATURE SURVEY}

Some research papers have examined the impact of routing protocols in VANET. Some concentrate on the connection between vehicle intensity and the routing protocol, while others focus on the particular constraints of the cars such as speed and speed. Three protocols are contrasted in [4][7]: OLSR, AODV and DYMO. The author has been compared. You find that AODV performs better than OLSR and DYMO in terms of products, but in terms of delay, DYMO is better than AODV. In this analysis, the transmission rate is relatively small. The research in [17][5] aims to find the impact on the efficiency of AODV and OLSR routing protocols of node density.Its results show that for low node density AODV improves throughput, while for high node density OLSR performs better. The key shortcoming of this research is the use of a mobility scenario that does not reflect real vehicle mobility (RandomWayPoint mobility pattern).

In [18][8] AODV and OLSR are replicated by Nakagami in the NS2 model. The results demonstrate that OLSR is stronger than AODV with a different node density in terms of packet transmission and throughput. But it seems that the difference is relatively small, particularly if the node intensity is high and the transmission rate impact is not measured in this analysis.

In [19][12] the authors assess the effects of the mobility model information in three VANET simulation case studies (especially traffic lights, the driver road choice, car overhaul behavior) and show that it is important for VANET Protocol design to select adequate level of detail in the simulation.

In the past, there have been some studies[20] on comparing the efficiency of VANET routing protocols. A comparison of the (AOMDV), Destination Sequenced Distance Vector (DSDV), Ad hoc on Demand Multipath Vectors is given on the basis of a practical simulation (AOMDV). A highly deteriorating urban realistic environment has been used for the simulated comparison of AODV and Optimized Link State (OLSR). The aim of associating the performance of protocols by [21] for the Routing of AODV, OLSR and DSDV is to use simple safety messages (BSM) and Average goodwill transferred 
from to each vehicle for simulations conducted with the highways image in the NS3 network simulator.

Data from the real maps was imported from [22][4] and [5] by the writers and tracks of the vehicle only in terms of actual road topology. In real life the flow of traffic in the city can be influenced by the rush hour and special events. There has been a real traffic generator designated, but traffic lights, highway speeds and more are not considered. Authors integrate VISSIM with the NS2 and enter a real traffic agreement, but VISSIM is not open source and thus this is not popular.

In this article, we are trying to answer the issue that traces of vehicles in a simulator cannot alter dynamically so that we can co-simulate NS3 and SUMO.

\section{METHODOLOGY}

In this article we use 2 files, 1 for (nodes.xml) and the other for (edge.xml) to translate these two files to the world (network.xml). then to link the nodes to the upper level vichers routes using a (sum.fy.xml) format. To transform a treac file into a (mobility.tcl) file, build a (triace.xml) file with the aid of (traceExporter.py). Usage of the success measure to evaluate the result in three senior citizens, the four protocols (OLSR, DSR, DSDV, AODV).

\subsection{NS3 and Sumo Feedback Loop}

With the TCP communication protocol between SUMO and NS3, we construct a feedback loop as shown in Figure 3.

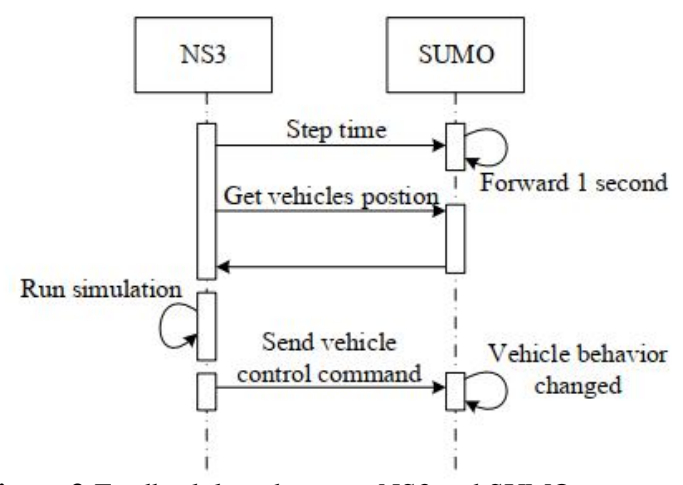

Figure 3:Feedback loop between NS3 and SUMO

The SUMO Time Frame uses a 'while loop' NS3. NS3 asks for the location of the vehicle in SUMO and updates it in SUMO each millisecond. After running the simulation protocol, NS3 adjusts its driving route by sending instructions dynamically in SUMO scenario. In reality, NS3 and SUMO are both established[23]-[25] and do not interact directly with dedicated experts. We use TraCI for our feedback loop in this article. TraCI is the default framework for linking road traffic and network simulators. traffic control interface. It is fitted with SUMO. We adapt a customer/server interactions model to complete our simulation when communication with both NS3 and SUMO. So, a customer we name TraciClient is introduced[26].
NS3 implements all layers supporting wireless communication for TraciClient and NS3, while TraciClient supports applications. If applications in this paper such as Change Route want to change the status of vehicles in SUMO, the messages from NS3 through TraciClient must be received first. With respect to TraciClient and SUMO interactions[27], TraciClient establishes applications-specific commands, then sends data to SUMO for execution and then retrieves SUMO data. In SUMO there is a TraciServer that scans these SUMO commands and then addresses the applications via TraciClient.

\subsection{Experiment}

The simulation was performed using the NS3 version 25 , combined through SUMO 25, as a highway transportation emulator, under the Ubuntu 14.04 operating system. Figure 4 and Figure 5shows Sumo web interface and traffic route. We utilize The Advanced heavily congested map.C++ was introduced \& developed in NS3 to assess the efficiency of the Protocols of Networking. The simulation is used to test the routing protocol output under different node densities (AODV, DSDV, OLSR, DSR) (20, 30,90). We utilize IEEE $802.11 \mathrm{p}$ as the data connection layer model for the simulation. We assume that all points in the system have a transmission capacity of 20 $\mathrm{dBm}$ and a transmission range of $145 \mathrm{~m}$.

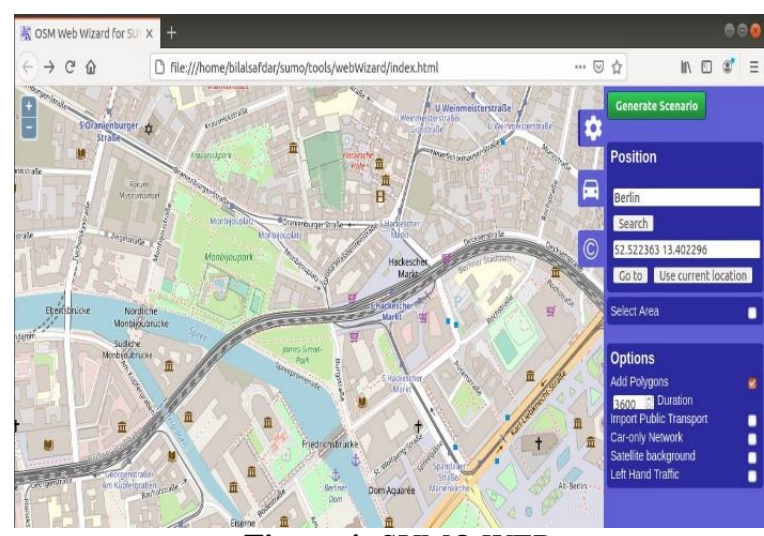

Figure 4: SUMO WEB

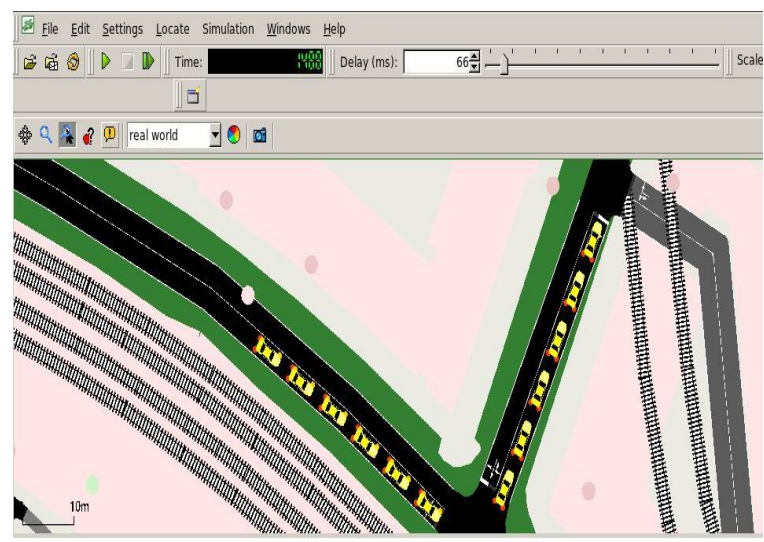

Figure 5: TrafficRoute 
C++ was introduced and developed into NS3 to assess the efficiency of the routing protocols. The simulation is used to test the routing protocol output under different node densities (AODV, DSDV, OLSR, DSR) (20, 30, 90).IEEE $802.11 \mathrm{p}$ used as the information connection level model for the simulation. We undertake that all nodes in the network have a transmission capacity of $25 \mathrm{dBm}$ and a broadcast array of $155 \mathrm{~m}$ which describe in table 1 .

Table 1:Parameters Values

\begin{tabular}{|l|l|}
\hline Parameters & Value \\
\hline Version of Simulator & NS3.25 \\
\hline Nodes Number & $25,30,35,50,80,90,100$ \\
\hline Simulation time & $120 \mathrm{sec}$ \\
\hline Area for Simulation & $1.7 * 1.7 \mathrm{~km}$ \\
\hline Size for the Packet & $256^{*} 2$ byte \\
\hline Type of Data & CB/R \\
\hline Name of Protocol & TCP \\
\hline Max Speed & $20 \mathrm{~m} / \mathrm{s}(40 \mathrm{~m} / \mathrm{s})$ \\
\hline Model used & TwoRayGround \\
\hline Channel/Layer & IEEE $(802.11 \mathrm{ah})$ \\
\hline Broadcast range & $155 \mathrm{~m}$ \\
\hline Broadcast power & $25 \mathrm{dBm}$ \\
\hline
\end{tabular}

\section{RESULTS AND DISCUSSION}

This is the ratio of the total information bits obtained and total data bits transmitted from sender to Receiver. PDR signifies the percentage of effective transmissions.

$$
P D R=\frac{\text { Packets Received }}{\text { Packets Transmitted }}
$$

Figure 6 shows that PDR increases in DSDV and OLSR routing protocols as node density increases.

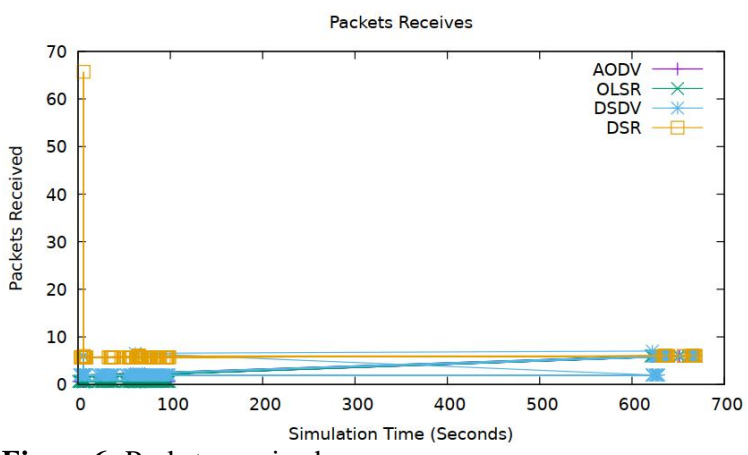

Figure 6: Packets received.

In this scenario DSDV protocols of routing, however, PDR is decreasing with an increase in density and is stable. The findings for DSDV and DSDV could be explained by the participation in the urban scenario of a large number of radio obstructions, thereby raising the probability of maximizing local problems.

Moreover, in perimeter mode the number of hops is increasing which can impact the PDR of DSDV and DSDV protocols negatively. In the case of OLSR, its efficiency much better than the other protocols. Sincethis one employ MPR structure that is useful for a higher and condensed network. Overhead refers to the extra bits inserted into the container, with the latest MAC and physically er messages, so that the recipient can understood the message as shown in Figure 7.

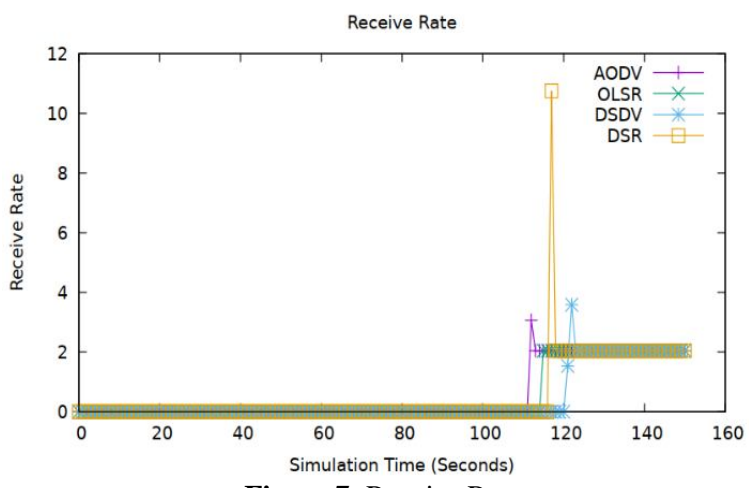

Figure 7: Receive Rate.

$$
\text { Overhead }=\frac{\text { totalPhyBytes-totalAppBytes }}{\text { totalPhyBytes }}
$$

Where the overhead is minimal, fewer bits than the actual data is requested for information, so that more information bits can clearly be directed into a packet.

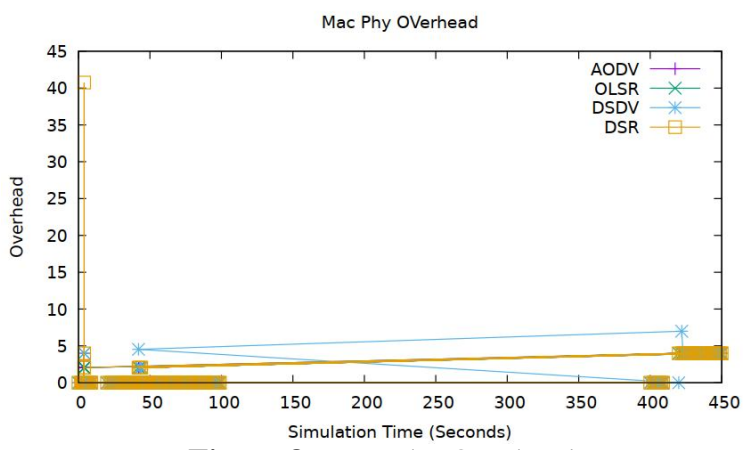

Figure 8: Mac Phy Overhead.

Above Figure 8 shows AODV is performing better at low node density than DSDV or OLSR, but DSDV is sunnier than AODV and OLSR when node compactness is increased. On behalf of AODV, outcome can be explicated which only requests a route while a node is sent, which creates a further delay when the number of nodes increases.

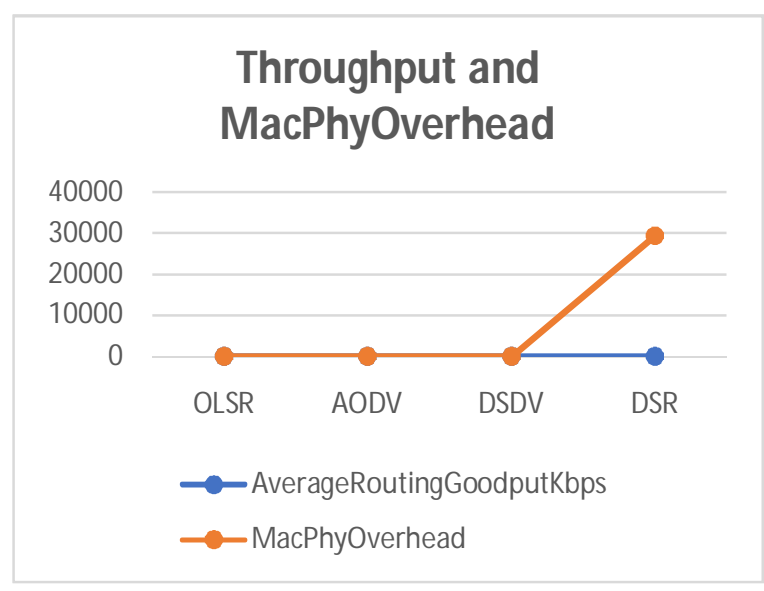

Figure 9:Throughput and MacPhyOverhead. 
Figure 9 shows the output, typically Refer to the total bytes correctly stored or transmitted, determined through kbps. If more data can be easily routed over through the network, routing protocols will enhance system performance.

Throughput $=\left(\frac{\text { Number of received packets }}{\text { Total Simulation Time }}\right) \times$ Packet Size

OLSR's transmission is stronger than DSDV and AODV, which drops as compactness increases. This can be explained by the fact that the DSDV transmissions the whole routing table after a fixed time period, which provides additional overhead and disturbs its performance. With $18 \mathrm{kbps}$ of OLSR, an MPR mechanism that reduces overhead routing could be used to achieve the result. In contrast to the other three protocols, DSR and DSR have the lowest possible performance as shown in Figure 10.

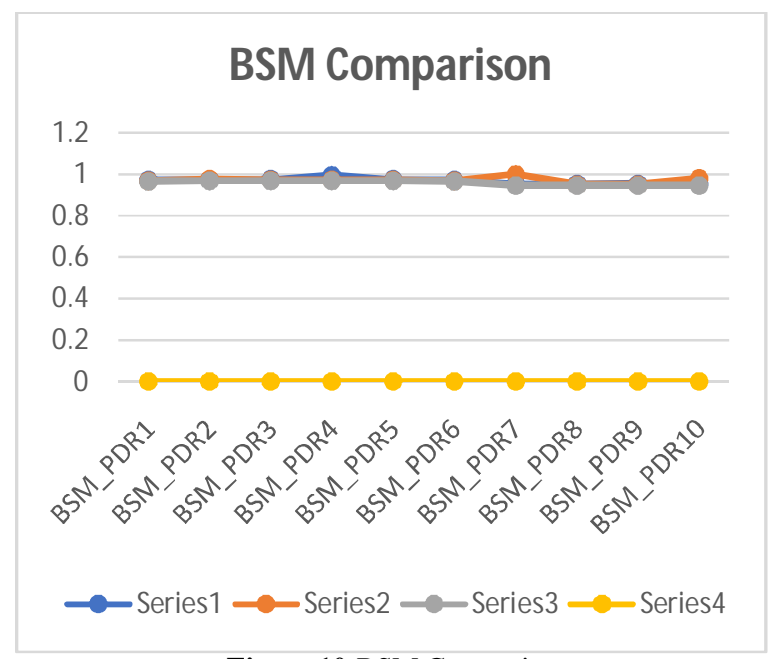

Figure 10:BSM Comparison.

The packet eliminated the requirement to wards usage of position package once again and decrease the upstairs of retransmitting nodes. The AODV has the ultimate overhead control since it provides a huge number of control packets in the direction of preserve the route.

\section{CONCLUSIONS}

The paper shows and evaluates the most popular VANET routing protocols with SUMO and NS-3 simulants to assess each algorithm's benefits and difficulties. OLSR's results in terms of PDR and performance could be regarded as the best performance for this consequence. In the case of overhead and E2E latency, AODV and DSDV are the finest two tracks. In fact, the destination location found through packet is determined by AODV and DSR to prevent the retransmitting nodes from re-using the locating service and reducing the overhead. In VANET, the field of protocol routing is a hot topic of research; many related problems must still be resolved.

\subsection{Future Work}

We will take a look at more metrics, such as the propagation model, the map size etc., that influence network output in the future. We trust that the instrument given for students and researchers on the field is useful and enjoyable.

\section{REFERENCES}

[1] Z. Cao, M. Diyan, and K. Han, A Routing Protocol Based on Multi-factor Decision in VANET,2020 IEEE Int. Conf. Consum. Electron. - Asia, ICCE-Asia 2020, pp. 14-17, 2020, doi: 10.1109/ICCE-Asia49877.2020.9277478.

[2] X. Guo, Y. Chen, L. Cao, D. Zhang, and Y. Jiang, A receiver-forwarding decision scheme based on Bayesian for NDN-VANET, China Commun., vol. 17, no. 8, pp. 106-120, 2020, doi: 10.23919/JCC.2020.08.009.

[3] P. Yellanki and M. V. S. P. Narasimham, Secure Routing Protocol for VANETS using ECC,2020 Int. Conf. Comput. Sci. Eng. Appl. ICCSEA 2020, pp. $\quad 0-4, \quad 2020, \quad$ doi: 10.1109/ICCSEA49143.2020.9132896.

[4] H. Yassine, VANET Cross-Layer Routing, pp. 1-2, 2019.

[5] S. Hu, Y. Jia, and C. She, Performance analysis of VANET routing protocols and implementation of a VANET terminal,Proc. 2017 Int. Conf. Comput. Technol. Electron. Commun. ICCTEC 2017, vol. 1, pp. 1248-1252, 2017, doi: 10.1109/ICCTEC.2017.00272.

[6] S. Yadav, N. K. Rajput, A. K. Sagar, and D. Maheshwari, Secure and reliable routing protocols for VANETs, 2018 4th Int. Conf. Comput. Commun. Autom. ICCCA 2018, pp. 1-5, 2018, doi: 10.1109/CCAA.2018.8777690.

[7] R. Kaur, M. Scholar, T. Pal, M. Singh, V. Khajuria, and M. Scholar, Network ( VANET ), no. Icoei, pp. 884-889, 2018.

[8] A. A. Celes and N. E. Elizabeth, Verification Based Authentication Scheme for Bogus Attacks in VANETs for Secure Communication,Proc. 2018 IEEE Int. Conf. Commun. Signal Process. ICCSP 2018, pp. 388392, 2018, doi: 10.1109/ICCSP.2018.8524540.

[9] W. Lai, H. Liu, and D. Tang, A contention window adaptation broadcast protocol in VANETs under differentiating transmission and virtual slot periods,Int. Conf. Commun. Technol. Proceedings, ICCT, vol. 2017-October, pp. 617-621, 2018, doi: 10.1109/ICCT.2017.8359710.

[10] A. Bhatia, K. Haribabu, K. Gupta, and A. Sahu, Realization of flexible and scalable VANETs through SDN and virtualization,Int. Conf. Inf. Netw., vol. 2018-January, pp. 280-282, 2018, doi: 10.1109/ICOIN.2018.8343125.

[11] E. R. Agustina and A. R. Hakim, Secure VANET protocol using hierarchical pseudonyms with blind signature,Proceeding 2017 11th Int. Conf. Telecommun. Syst. Serv. Appl. TSSA 2017, vol. 2018-January, pp. 1-4, 2018, doi: 10.1109/TSSA.2017.8272919.

[12] H. Kaur and Meenakshi, Analysis of VANET geographic routing protocols on real city map,RTEICT 2017 - 2nd IEEE Int. Conf. Recent Trends Electron. Inf. Commun. Technol. Proc., vol. 2018-January, pp. 895-899, 2017, doi: 10.1109/RTEICT.2017.8256727.

[13] S. K. Singh, Performance evaluation of beacons 
control data dissemination protocol in handover scenario for VANET,Proc. - 2017 IEEE Int. Conf. Electr. Instrum. Commun. Eng. ICEICE 2017, vol. 2017-December, pp. 1-6, 2017, doi: 10.1109/ICEICE.2017.8191954.

[14] M. Syfullah and J. M. Y. Lim, Data broadcasting on Cloud-VANET for IEEE 802.11p and LTE hybrid VANET architectures,3rd IEEE Int. Conf. , pp. 1-6, 2017, doi: 10.1109/CIACT.2017.7977321.

[15] V. Nguyen, O. T. T. Kim, T. N. Dang, and C. S. Hong, Improving time slot acquisition through RSU's coordination for TDMA-based MAC protocol in VANETs,Int. Conf. Inf. Netw., vol. 2016-March, pp. 406-411, 2016, doi: 10.1109/ICOIN.2016.7427145.

[16] G. Farrokhi and S. Zokaei, Improving safety message dissemination in IEEE 802.11e based VANETs using direction oriented controlled repetition technique,Proc. 2014 IEEE 21st Symp. Commun. Veh. Technol. BeNeLux, IEEE SCVT 2014, pp. 100-104, 2014, doi: 10.1109/SCVT.2014.7046716.

[17] V. Kolici, T. Oda, E. Spaho, L. Barolli, M. Ikeda, and K. Uchida, Performance Evaluation of a VANET Simulation System Using NS-3 and SUMO,Proc. - IEEE 29th Int. Conf. Adv. Inf. Netw. Appl. Work. WAINA 2015, pp. 348-353, 2015, doi: 10.1109/WAINA.2015.121.

[18] G. Sallam and A. Mahmoud, Performance Evaluation of OLSR and AODV in VANET Cloud Computing Using Fading Model with SUMO and NS3,2015 Int. Conf. Cloud Comput. ICCC 2015, pp. 1-5, 2015, doi: 10.1109/CLOUDCOMP.2015.7149649.

[19] T. S. Chouhan and R. S. Deshmukh, Analysis of DSDV, OLSR and AODV Routing Protocols in VANETS Scenario: Using NS3, Proc. - 2015 Int. Conf. Comput. Intell. Commun. Networks, CICN 2015, pp. 85-89, 2016, doi: 10.1109/CICN.2015.26.

[20] F. Benabdallah, A. Hamza, and M. Bechrif, Simulation and analysis of VANETS performances based on the choice of mobility model,Proc. Comput. Conf. 2017, vol. 2018January, no. July, pp. 1238-1242，2018， doi: 10.1109/SAI.2017.8252248.

[21] A. M. Shaban, S. Kurnaz, and A. M. Shantaf, Evaluation DSDV, AODV and OLSR routing protocols in real live by using SUMO with NS3 simulation in VANET,HORA 2020 - 2nd Int. Congr. Human-Computer Interact. Optim. Robot. Appl. Proc., pp. 6-10, 2020, doi: 10.1109/HORA49412.2020.9152903.

[22] R. Aji Pratama, L. Rosselina, D. Sulistyowati, R. Fitri Sari, and R. Harwahyu, Performance Evaluation on VANET Routing Protocols in the Way Road of Central Jakarta using NS-3 and SUMO,Proc. - 2020 Int. Semin. Appl. Technol. Inf. Commun. IT Challenges Sustain. Scalability, Secur. Age Digit. Disruption, iSemantic 2020, pp. 280-285, 2020 , 10.1109/iSemantic50169.2020.9234202.
[23] J. Lyu, C. Chen, and H. Tian, Secure Routing Based on Geographic Location for Resisting Blackhole Attack in Three-dimensional VANETs,2020 IEEE/CIC Int. Conf. Commun. China, ICCC 2020, no. Iccc, pp. 1168-1173, 2020, doi: 10.1109/ICCC49849.2020.9238997.

[24] W. Liu, X. Wang, W. Zhang, L. Yang, and C. Peng, Coordinative simulation with SUMO and NS3 for Vehicular Ad Hoc Networks, Proc. Asia-Pacific Conf. Commun. APCC 2016, pp. 337-341, 2016, doi: 10.1109/APCC.2016.7581471.

[25] M. S. M. Shihab, A. A. Ibrahim, and A. M. Shantaf, Evaluate the DSR and OLSR Routing Protocols in different scenarios under NS3 and SUMO Using the NETEDITOR in VANETs, 4 th Int. Symp. Multidiscip. Stud. Innov. Technol. ISMSIT 2020 - Proc., pp. 1-5, 2020, doi: 10.1109/ISMSIT50672.2020.9254338.

[26] B. Amina and E. Mohamed, Performance Evaluation of VANETs Routing Protocols Using SUMO and NS3,Colloq. Inf. Sci. Technol. Cist, vol. 2018-October, pp. 525-530, 2018, doi: 10.1109/CIST.2018.8596531.

[27] S. I. Chowdhury, W. Il Lee, Y. S. Choi, G. Y. Kee, and J. Y. Pyun, Performance evaluation of reactive routing protocols in VANET, 17th AsiaPacific Conf. Commun. APCC 2011, no. October, pp. 559-564, 2011, doi: 10.1109/APCC.2011.6152871. 